\title{
Haematological monitoring for clozapine: do patients know why?
}

\author{
Carol Paton and Paul Wolfson
}

\begin{abstract}
Clozapine is effective in treatment resistant schizophrenia, but unfortunately is associated with o $3 \%$ incidence of neutropenia. Regular hoematological monitoring is mandatory for all patients. We asked forty patients who had been taking clozapine for more than six months why they thought they had to hove regular blood tests. Almost half did not know and only a small proportion were subject to a Mental Health Act (MHA) second opinion for consent to treatment. Initial explanations of the potentially serious side effects of clozapine may not be understood or retained. Ongoing education of patients is essential. The wider use of MHA second opinion doctors should also be considered.
\end{abstract}

Over 6500 patients in the UK currently receive clozapine and this number is increasing [Clozapine Patient Monitoring Service [CPMS], personal communication). The drug is associated with a $3 \%$ incidence of neutropenia and in spite of strict haematological monitoring coordinated by the CPMS, there have been two deaths associated with bone marrow suppression in the UK. Informed consent, a complex issue in patients with severe and long-standing mental illness (Jeffries, 1993), is therefore particularly relevant when offering treatment with clozapine. We sought to investigate how informed patients who had been taking clozapine for more than six months were about its potentially fatal side effect, and how their degree of understanding related to the severity of residual illness, clinical response to clozapine and current Mental Health Act status.

\section{The study}

Using the pharmacy computer link with the CPMS, we identified all patients within a provider unit who had been taking clozapine for longer than six months. Patients who consented to be interviewed as part of a more general inquiry about their views on clozapine were asked by a pharmacist why they thought they had regular blood tests. The initial open question was supplemented by further directed inquiries in those who gave an inadequate or delusional explanation. The Mental Health Act (MHA) status of the patients was recorded.

We classified explanations as adequate if patients understood that clozapine may have a serious effect on the composition of their blood which could make them very ill. Patients who demonstrated some understanding of the issues, but did not fully grasp their implications, were classified separately from those who had no understanding.

In order to determine the patient's current severity of illness, the Brief Psychiatric Rating Scale (BPRS; Overall \& Gorham, 1962) was administered by a psychiatrist. This was used in conjunction with the opinions of nursing staff and relatives in order to complete the Clinical Global Severity (CGS; Guy, 1976). In the absence of BPRS scores prior to starting clozapine the Clinical Global Impression of Improvement (CGI; Guy, 1976) was rated on the basis of the opinions of nursing staff and relatives.

\section{Findings}

We identified 40 patients, of whom 37 consented to be interviewed. Eight (22\%) gave an adequate explanation of why they were having regular blood tests, ten (27\%) demonstrated partial understanding, 18 (49\%) no understanding and one patient was unable to cooperate with the interview procedure. Examples of explanations demonstrating partial or no understanding are shown in Table 1.

Out-patients were no more likely to understand than informal or detained inpatients. Of those patients showing no understanding three were out-patients, seven informal in-patients, four detained patients who were consenting to treatment and the remaining four were detained patients who had a valid Form 39 completed by a second opinion doctor under section 58 of the MHA (1983). The BPRS scores of this group of 
Table 1. Examples of explanations demonstrating partial understanding and no understanding of the rationale behind haematological monitoring for clozapine

Explanations demonstrating partial understanding

To do with blood cells, but I'm not sure what.

It concerns white corpuscles, so l've been told, but I don't know why it should concern them, I just don't know.

Something to do with white, but white what I don't

know. White is the key to it all.

It's to do with blood cells.

in case anything goes wrong.

Explanations demonstrating no understanding

Because the doctor says so.

I've often wondered the same thing, but I need them to take the tablets, it's very important.

It's to look in my blood so that the pharmacy can find the best thing to cure me.

Check the level, or is that the lithium. No, I've no idea at all. Tell me.

To see if I've been on drugs, cannabis and that.

To check if I've been taking the tablets.

To see if it's been working.

There's no reason for it at all, it's all gone too far

can't really say.

For research, isn't it.

To check the blood level.

It's because my tablets are yellow.

To analyse the quality-rich or anaemic and keep down the white corpuscles.

Because I have to.

I refuse to talk about this subject without my lawyer.

To see if there is any sugar in my blood or if I have AIDS.

Helps to thin it and keep me healthy.

Blood letting because I've been doped.

patients on the 18 item schedule ranged from 4-46 (mean 23, standard deviation 9). These scores, in conjunction with the opinions of nursing staff and relatives, identified one patient as borderline mentally ill, one as mildly ill, nine as moderately ill, two as markedly ill, four as severely ill and one as being among the most severely ill patient on the CGS scale. The opinions of nursing staff and relatives identified 12 of these patients as having very much or much improved on the CGI. A further four had minimally improved and there was no change in the remaining two.

\section{Comment}

Although the patients had received clozapine for more than six months, approximately half of them had no understanding of the risks of treatment and were therefore unable to give informed consent.

A minority of the patients were detained under the MHA and had a current Form 39. The Mental Health Act Commission guidelines (1993) state that patients frequently respond rapidly to clozapine and that the low incidence of side-effects facilitates full and rapid consent to treatment. Our findings, however, suggest that while patients may well cooperate with haematological monitoring, they do not fully understand its purpose. Difficulties with informed consent in patients with severe and long standing mental illness are not restricted to clozapine. How many psychiatrists can confidently state that all, or even the majority of informal patients under their care, for whom they prescribe conventional antipsychotics, understand the risks and implications of tardive dyskinesia?

Treatment with clozapine is often started when patients are acutely symptomatic, and it is likely that explanations provided at the time by staff would be poorly absorbed or retained. At this point it would be prudent to record in the case-notes a patient's understanding of the risks involved. Up to $60 \%$ of treatment resistant patients can be expected to respond to clozapine (Kane, 1992) and the results of our study are in line with these findings. It would be unethical as well as impractical to detain all patients under the Mental Health Act who have responded to treatment but do not understand the risks involved. However, our study does suggest that there is a need for the wider use of second opinion doctors when detained patients are treated with clozapine. Additionally, there is a need for on-going education for all patients about the risks involved in order that they can give informed consent. The relatively high incidence of a potentially fatal side effect should make these issues a concern for all prescribers of clozapine.

\section{References}

GUY, W. (1976) ECDEU Assessment Manual for Psychopharmacology, revised DHEW Pub. No. (ADM) 78-388. Rockville, MD: National Institute of Mental Health. JEFFRIES, J. J. (1993) Ethical issues in drug selection for schizophrenia. Canadian Journal of Psychiatry. 38 (suppl. 3), 70-74.

KANE. J. M. (1992) Clinical efficacy of clozapine in treatment-refractory schizophrenia: an overview. British Journal of Psychiatry. 160 (suppl. 17), 41-45.

MENTAL HEALth ACT Commission (1993) Guidance on the administration of clozapine and other treatments requiring blood tests under the prouistons of part IV of the Mental Health Act. Practice note 1.

OVERAL., J. E. \& Gorham, D. R. (1962) Brlef Psychiatric Rating Scale. Psychological Reports, 10, 799-812.

*Carol Paton, Pharmacist, and Paul Wolfson, Consultant Psychiatrist, Bexdey Hospital, Old Bexley Lane, Bexley, Kent DA5 2BW

*Correspondence 